\title{
Prädiktor für das Nichtansprechen auf eine antidepressive Therapie
}

Fragestellung: Eignet sich eine Hyperaktivität im Subcallosalen Zingulum (SCC) als Prädiktor für das Nichtansprechen auf eine antidepressive Kombinationstherapie aus kognitiver Verhaltenstherapie plus Escitalopram?

Hintergrund: Nur etwa ein Drittel der Patienten mit Major Depression erleben eine Remission der Symptomatik beim ersten Behandlungsversuch. War der erste Behandlungsversuch nicht erfolgreich, erfolgt in der Regel der nächste Behandlungsschritt. Das Umstellen der Antidepressiva oder die Kombination oder Augmentation des Medikaments erhöht die Remissionsrate um weitere $15-20 \%$, allerdings erleben etwa $20-30 \%$ der Patienten auch nach mehreren Behandlungsversuchen keine vollständige Remission. Ein geeigneter Biomarker soll dabei helfen, vor Therapiebeginn festzustellen, ob die geplante Therapie erfolgreich sein wird oder nicht. Eine Hyperaktivität des SCC wurde wiederholt mit dem Ausbleiben eines positiven Therapieansprechens assoziiert. Die SCC-Aktivierung könnte daher ein in der Therapiesteuerung einsetzbarer Prädiktor sein.

Patienten und Methodik: Die Studie bestand aus zwei Therapieschritten: Vor Behandlungsbeginn wurden 82 Patienten mit Major Depression auf eine zwölfwöchige Monotherapie mit Escitalopram oder kognitiver Verhaltenstherapie (KVT) randomisiert. Sie erhielten in den ersten drei Behandlungswochen $10 \mathrm{mg}$ Escitalopram pro Tag, da-

McGrath CL, Kelley ME, Dunlop BW et al. Pretreatment brain states identify likely nonresponse to standard treatments for depression. Biol Psychiatry 2014; 76: $527-35$ nach $20 \mathrm{mg} / \mathrm{Tag}$. Die KVT bestand im ersten Behandlungsabschnitt aus 16 Einzelsitzungen. Im zweiten Abschnitt wurde einmal im Monat eine Booster-Sitzung durchgeführt. Nach Ab- schluss der ersten Therapiephase erhielten Nonremitter (HAMD $>$ 7) für weitere zwölf Wochen eine Kombinationstherapie aus Escitalopram plus KVT. Die Depressionsschwere wurde von verblindeten Ratern wöchentlich mit der Hamilton Depression Rating Scale (HAMD17) erfasst. Vor Studienbeginn erhielten alle Teilnehmer ein FDG-PET. Hauptzielregion war der SCC. Zudem wurde eine Whole-brain-Analyse vorgenommen. Untersucht wurde, ob sich die Hirnaktivierung der Remitter (Step 1 oder 2) vor Behandlungsbeginn von der Hirnaktivierung der Nonresponder (Veränderung der Depressionsschwere < $50 \%$ ) unterschied. Zusätzlich wurden 24 gesunde Kontrollen untersucht.

Ergebnisse: Im ersten Behandlungsabschnitt waren 23 Patienten Remitter, 30 zeigten keine Remission und wurden in den zweiten Behandlungsabschnitt aufgenommen. Nach 24 Wochen remittierten zwölf weitere Patienten. Diese wurden mit neun Nonrespondern verglichen. Hypothesenkonform war der Metabolismus des SCC vor Behandlungsbeginn bei Nonrespondern höher als bei Remittern oder Gesunden. Remitter und gesunde Kontrollprobanden unterschieden sich in ihrer SCC-Aktivierung nicht voneinander. Zusätzlich zeigten die Analysen bei Nonrespondern eine höhere Aktivierung im rechten superioren Temporalsulcus, die Aktivierung der Remitter war jedoch geringer als die der gesunden Teilnehmer. Die Hirnaktivierung von Patienten, die im ersten Therapieabschnitt Escitalopram oder KVT erhielten, unterschied sich nicht voneinander, ebenso wenig wie die der Remitter des ersten und zweiten Behandlungsabschnitts.

Schlussfolgerungen: Die Ergebnisse legen nahe, dass eine Überaktivierung im SCC ein Hauptkriterium von Patienten ist, die nicht auf Escitalopram oder KVT ansprechen. Diese Patienten sollten laut der Autoren daher nicht mit einem SSRI oder KVT behandelt werden.

\section{- Kommentar von Stefanie Wagner und André Tadić, Mainz}

\section{Vielversprechender Prädiktor für Depressionen identifiziert?}

Die Studie hatte das Ziel, einen Prädiktor für das Ansprechen auf eine antidepressive Therapie zu identifizieren. Sie kommt zu dem Ergebnis, dass Patienten mit Hyperaktivität im SCC im ersten Schritt nicht mit einem SSRI oder KVT behandelt werden sollten. Aufgrund der wenig zufriedenstellenden Remissionsraten depressiver Patienten im ersten Therapieversuch wäre die Identifikation eines Markers für Pharmakotherapieresistenz von hoher klinischer und ökonomischer Relevanz. Die Anzahl der Nonresponder in der Studie war sehr gering und der Marker muss in unabhängigen Studien repliziert werden, bevor weitere Studien erfolgen. Welche Therapie für diese mögliche Risikogruppe dann geeignet wäre, ist noch nicht abzusehen.

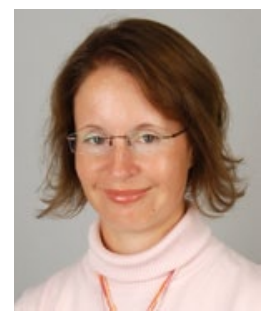

Dr. hum. biol. Stefanie Wagner, Mainz

Klinik für Psychiatrie und Psychotherapie, Universitätsmedizin Mainz E-Mail: stefanie.wagner@unimedizin-mainz.de 